\title{
Efficacy and safety of sphincterotomy with sphincteroplasty using large caliber balloons in the treatment of choledocholithiasis with extraction difficulties
}

\author{
R. Uribarrena-Amezaga ${ }^{1}$, I. Aured De La Serna ${ }^{1}$, I. Calvo-Morillas ${ }^{1}$, J. J. Sebastián-Domingo ${ }^{2}$, \\ T. Cabrera-Chaves ${ }^{2}$, M. T. Soria San Teodoro ${ }^{1}$, R. Uribarrena-Echebarría ${ }^{1}$ \\ ${ }^{1}$ Department of Digestive Diseases, Miguel Servet University Hospital, Zaragoza, Spain \\ ${ }^{2}$ Department of Digestive Diseases, Royo Villanova Hospital, Zaragoza, Spain \\ Email: uribarrena@hotmail.com
}

Received 9 June 2013; revised 11 July 2013; accepted 25 July 2013

Copyright (c) 2013 R. Uribarrena-Amezaga et al. This is an open access article distributed under the Creative Commons Attribution License, which permits unrestricted use, distribution, and reproduction in any medium, provided the original work is properly cited.

\begin{abstract}
Introduction: Endoscopic retrograde cholangiopancreatography (ERCP) with endoscopic sphincterotomy (ES) is the most widely used technique for treating choledocholithiasis. In some cases, due to anomalies of the papilla or the presence of large or multiple calculi, additional maneuvers are needed to remove the stones. The present study investigates the efficacy and safety of ES with sphincteroplasty (SP) in the management of choledocholithiasis with extraction difficulties. Patients and Methods: A prospective study was made of 153 patients with choledocholithiasis subjected to ERCP. Fifty-two patients underwent ES with SP, while 101 were subjected only to ES. The two groups were compared in terms of age, gender, percentage cannulation, presence of papilla alterations, large or multiple stones, success in stone removal and complications (acute pancreatitis, bleeding and perforation). In the ES with SP group, we moreover recorded the diameters of the balloons employed (10 - 18 mm). Results: There were no significant differences between the groups in the stone extraction success rate $\mathbf{9 4 . 2 3 \%}$ in the ES with SP group versus $97.03 \%$ in the ES group) or in the appearance of complications (3.8\% in the ES with SP group versus $2.7 \%$ in the ES group). The presence of difficult papillae, and of multiple or large stones was significantly greater in the ES with SP group. The diameter of the balloon was not associated with the appearance of complications. Conclusion: Endoscopic sphincterotomy with sphincteroplasty is effective and safe in the treatment of choledocholithiasis with extraction difficulties.
\end{abstract}

Keywords: Choledocholithiasis; Endoscopic

Sphincterotomy; Sphincteroplasty

\section{INTRODUCTION}

Endoscopic retrograde cholangiopancreatography (ERCP) with endoscopic sphincterotomy (ES) is the most widely used technique for treating choledocholithiasis (CL) [1,2]. However, a number of factors may greatly complicate the extraction of the stones. Large or multiple stones and certain alterations of the papilla that impede extensive ES are some of the circumstances that most often complicate success of the procedure [3]. In the past, mechanical lithotripsy was more often used, and if this technique failed, surgery was decided. In elderly patients or individuals with a high surgical risk, the placement of plastic biliary stents is an acceptable alternative [4]. However, the devices used for mechanical lithotripsy are often complicated to assemble and handle. Furthermore, the technique is not without complications [5]. The surgical option is more aggressive and involves greater morbidity and mortality than endoscopic treatment, particularly in very elderly individuals, which are the typical type of patients that suffer CL. For these reasons, sphincteroplasty (SP) with dilating balloons was introduced in 2003 as a routine method for expanding endoscopic sphincterotomies that are found to be insufficient. It was made of the same balloons designed for dilating stenotic zones in other sections of the gastrointestinal tract, with calibers ranging between 10 and $20 \mathrm{~mm}$ [6]. The present study was carried out to evaluate the efficacy and safety of the combination of ES with SP in our hands for the treatment of choledocholithiasis with extraction difficul- 
ties, and to determine whether the complications are greater than when only ES is performed (Figure 1).

\section{PATIENTS AND METHODS}

\subsection{Patients and Procedure}

A prospective study was made of 153 patients diagnosed with choledocholithiasis in Miguel Servet University Hospital (Zaragoza, Spain) during the period between January 2009 and November 2011. All of the patients were subjected to ERCP with ES. Fifty-two of them moreover underwent SP after ES, and were compared with the 101 patients of the control group, subjected only to ES. The indication of one technique or other depended on the criterion of the endoscopist, after evaluating the difficulty of stone removal. Written informed consent was obtained from all patients at least 24 hours in advance. Antibiotic prophylaxis in the form of intravenous amoxicillin-clavulanate was provided according to the protocol used in our hospital, unless the patient had already been receiving broader spectrum antibiotics. In patients with allergy to betalactams, amoxicillin was replaced with ciprofloxacin plus metronidazole. The procedure was carried out under sedation with midazolam and intravenous fentanyl, administered by the endoscopist, with the monitorization of oxygen saturation and heart rate. Intravenous buscapine and atropine were also administered. The canulation was performed with 0.025

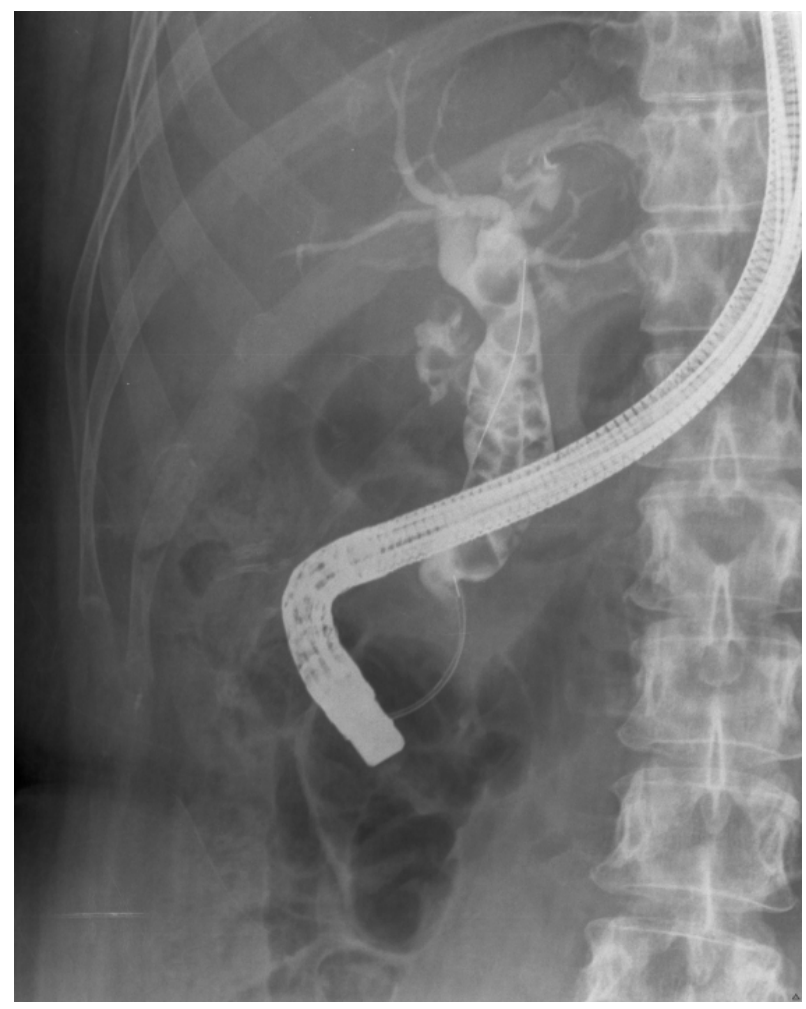

Figure 1. Multiple and big bile duct stones. and 0.035 inch guidewire double lumen sphincterotomes (Olympus $^{\circledR}$ ). If direct canulation was not possible, we used a needle knife (Olympus ${ }^{\circledR}$ ) to make a precut. In performing SP we used progressive 10 - $18 \mathrm{~mm}$ diameter balloons, of the kind commonly used to dilate stenotic zones in other sections of the gastrointestinal tract (Boston Scientific $\left.{ }^{\circledR}\right)$. Dilatation diameter and pressure was controlled by a manometer and maintained between 20 and 30 seconds for each caliber, and no more than three different measures were used in each case. Removal of the stones was carried out with a Fogarty balloon or Dormia basket. In no case were pancreatic stents used.

The procedures were carried out by two endoscopists experienced with the technique (Figures 2 and $\mathbf{3}$ ).

Data were collected related to the following:

Patients: age and gender.

Technique: need for precut, performance of SP after

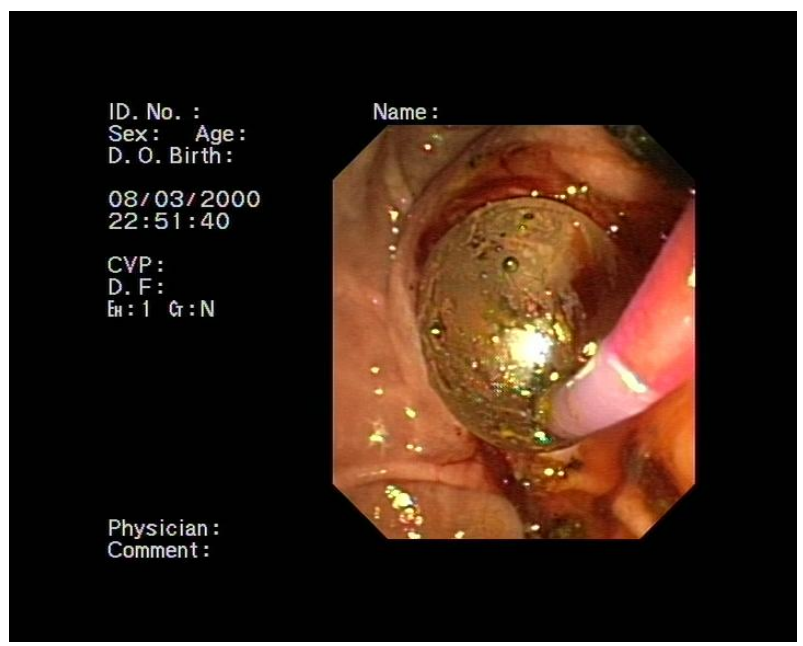

Figure 2. Sphincterotomy and sphinteroplasty with large caliber balloon.

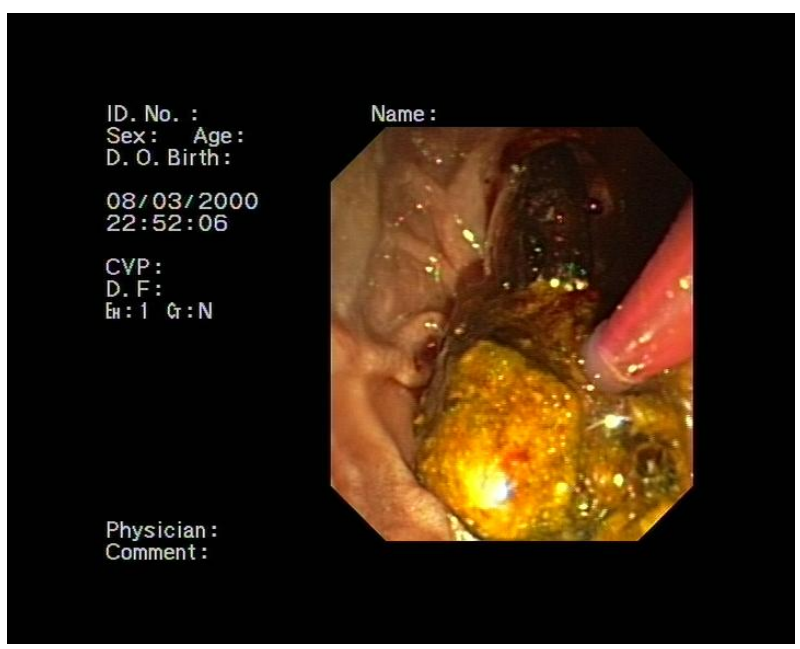

Figure 3. Bile duct stones extracción after sphincterotomy plus sphincteroplasty. 
ES, and caliber of the dilatation balloons.

Papilla: a difficult or risk papilla in relation to ES was defined by the presence of dissimilarity between the size of the papilla and the diameter of the stones, intradiverticular papillae, and patients with previous ES.

Stones: multiple (more than 2 ) or large ( $>10 \mathrm{~mm}$ in diameter).

Complications: acute pancreatitis, perforation, significant digestive bleeding, cholangitis or acute cholecystitis and death. Acute pancreatitis and its severity were defined following the criteria of Cotton [7,8].

We only registered significant bleeding requiring transfusion or endoscopic or surgical hemostatic maneuvering after the procedure. Mild or self-limited bleeding requiring no special measures and implying no prolongation of hospital stay were not considered.

Success was taken to represent complete removal of the stones, while failure was considered when otherwise. In the event of failure, we resorted to biliary stent placement consumables or surgery.

\subsection{Statistical Analysis}

\subsubsection{Descriptive Analysis}

In the descriptive analysis qualitative variables were expressed as percentages. In the case of quantitative variables, we first evaluated normal distribution with the Kolmogorov-Smirnov and Shapiro-Wilks tests. Those quantitative variables exhibiting a normal distribution were reported as the mean and standard deviation, while variables with a non-normal distribution were reported as the mean, median and interquartile range.

\subsubsection{Inferential Analysis}

The following statistical tests were used for the simple or bivariate analyses:

$>$ Pearson chi-squared test: This test was used for the comparison of two categorical variables. As criterion for using this test, all the expected values in the contingency table were required be greater than 5 .

> Fisher exact test: This test was used for the comparison of two dichotomic categorical variables when all the expected values in the contingency table were over 1 and one or more were under 5 .

> Linear association test: This test was used for the comparison of categorical variables in which one variable was of an ordinal qualitative nature with more than two categories.

$>$ Student t-test: This test was used for the comparison of two means corresponding to a quantitative variable versus a dichotomic variable. As criterion for applying the Student t-test, the quantitative variable was required to present a normal distribution for each of the sample subgroups defined by the values which the dichotomic variable may present.

> Mann-Whitney U-test: This nonparametric test was used for the comparison of two means when the criteria required for applying the Student t-test were not met.

Assessment of the magnitude of the association was based on calculation of the hazard ratio or relative risk (RR), while the precision of the estimation of effect was measured using the $95 \%$ confidence interval (CI) of the RR.

Statistical significance was considered for $\mathrm{p}<0.05$.

\section{RESULTS}

A total of 153 patients (77 males and 66 females) between 18 and 97 years of age (mean 74.23 years, standard deviation (SD) 13.8) were included in the study. The mean age of the ES with SP group (75.25 years, SD 12.55) was similar to that of the ES group (73.71 years, SD 14.44). As regards the gender distribution, males and females respectively represented $42.31 \%$ and $57.69 \%$ in the ES with SP group, and $54.46 \%$ and $45.54 \%$ in the ES group.

Bile duct cannulation was achieved in all patients: directly in 150 cases (98.08\% in the ES with SP group and $98.02 \%$ in the ES group) and using precut in three cases (1 in the ES with SP group and 2 in the ES group).

We used balloons with calibers between 10 and $18 \mathrm{~mm}$, depending on the characteristics of the papilla, the size and number of stones, and the bile duct diameter. The mean balloon diameter was $12.80 \mathrm{~mm}$ (SD 2.28) (Descriptive quantitative and qualitative variable are summarized in Tables $\mathbf{1}$ and 2).

The procedure proved successful in 49 of the 52 patients in the ES with SP group (94.23\%) and in 98 of the 101 patients of the ES group (97.03\%). The difference between the two groups was not statistically significant $(p=0.409)$ (Table 3). In the 6 patients in which stone removal was not possible, we placed between one and three plastic stents (caliber $10 \mathrm{Fr}$ ) in 5 cases. One patient in the ES with SP group required emergency surgery due to perforation with retro-pneumoperitoneum and stone impaction in the distal choledochus.

The presence of a difficult or risk papilla for ES was significantly more frequent in the ES with SP group $(76.92 \%)$ than in the ES group (36.63\%) $(\mathrm{p}=0.000)$. Likewise, stones measuring over $1 \mathrm{~cm}$ in size were more common in the ES with SP group than in the ES group ( $50 \%$ versus $26.73 \%$, respectively; p 0.001 ), in the same way as the presence of multiple stones (38.46\% versus 14.84\%; p 0.007).

The global percentage of relevant complications was $3.28 \%$ (5 out of 153 cases). The most frequent problem was acute pancreatitis ( 3 cases, 1.98\%). In no case was the latter serious, however. There were no deaths related to the technique.

Analyzed by groups, the patients subjected to ES with 
Table 1. Descriptive study. Qualitative variables in ES + SP and ES groups.

\begin{tabular}{|c|c|c|c|c|c|}
\hline & & \multicolumn{4}{|c|}{ Case or control } \\
\hline & & \multicolumn{2}{|c|}{ Case (ES + SP group) } & \multicolumn{2}{|c|}{ Control (ES group) } \\
\hline & & $\mathrm{N}$ & $\%$ & $\mathrm{~N}$ & $\%$ \\
\hline \multirow{2}{*}{ Gender } & Male & 22 & $42.31 \%$ & 55 & $54.46 \%$ \\
\hline & Female & 30 & $57.69 \%$ & 46 & $45.54 \%$ \\
\hline \multirow{2}{*}{ Difficult papilla } & Yes & 40 & $76.92 \%$ & 37 & $36.63 \%$ \\
\hline & No & 12 & $23.08 \%$ & 64 & $63.37 \%$ \\
\hline \multirow{2}{*}{ Diverticulum } & Yes & 14 & $26.92 \%$ & 29 & $28.71 \%$ \\
\hline & No & 38 & $73.08 \%$ & 72 & $71.29 \%$ \\
\hline \multirow{2}{*}{ Small size } & Yes & 10 & $19.23 \%$ & 2 & $1.98 \%$ \\
\hline & No & 42 & $80.77 \%$ & 99 & $98.02 \%$ \\
\hline \multirow{2}{*}{ Previous EE } & Yes & 17 & $32.69 \%$ & 5 & $4.95 \%$ \\
\hline & No & 35 & $67.31 \%$ & 96 & $95.05 \%$ \\
\hline \multirow{2}{*}{ Large stone } & Yes & 26 & $50.00 \%$ & 27 & $26.73 \%$ \\
\hline & No & 26 & $50.00 \%$ & 74 & $73.27 \%$ \\
\hline \multirow{2}{*}{ Multiple stones } & Yes & 20 & $38.46 \%$ & 15 & $14.85 \%$ \\
\hline & No & 32 & $61.54 \%$ & 86 & $85.15 \%$ \\
\hline \multirow{2}{*}{ Perforation } & Yes & 1 & $1.92 \%$ & 0 & $0.00 \%$ \\
\hline & No & 51 & $98.08 \%$ & 101 & $100.00 \%$ \\
\hline \multirow{2}{*}{ Bleeding } & Yes & 0 & $0.00 \%$ & 1 & $0.99 \%$ \\
\hline & No & 52 & $100.00 \%$ & 100 & $99.01 \%$ \\
\hline \multirow{2}{*}{ Pancreatitis } & Yes & 1 & $1.92 \%$ & 2 & $1.98 \%$ \\
\hline & No & 51 & $98.08 \%$ & 99 & $98.02 \%$ \\
\hline \multirow{2}{*}{ Death } & Yes & 0 & $0.00 \%$ & 0 & $0.00 \%$ \\
\hline & No & 52 & $100.00 \%$ & 101 & $100.00 \%$ \\
\hline \multirow{2}{*}{ Type of access } & Direct cannulation & 51 & $98.08 \%$ & 99 & $98.02 \%$ \\
\hline & Precut & 1 & $1.92 \%$ & 2 & $1.98 \%$ \\
\hline \multirow{2}{*}{ Extraction of stones } & Yes & 49 & $94.23 \%$ & 98 & $97.03 \%$ \\
\hline & No & 3 & $5.77 \%$ & 3 & $2.97 \%$ \\
\hline
\end{tabular}

Table 2. Descriptive study. Quantitative variables in ES + SP and ES groups.

\begin{tabular}{ccccccc}
\hline & \multicolumn{1}{c}{ Total } & \multicolumn{3}{c}{ Cases (ES + SP) } & \multicolumn{3}{c}{ Controls (ES) } \\
\hline & Mean & SD & Mean & SD & Mean & SD \\
Age & 74.23 & 13.80 & 75.25 & 12.55 & 73.71 & 14.44 \\
Balloon diameter & 12.80 & 2.28 & 12.80 & 2.28 & & \\
\hline
\end{tabular}

SP suffered two major complications (3.8\%): pancreatitis in one case (1.9\%) and perforation at papillary level during extraction maneuvering in another case (1.9\%). In the ES group we recorded three complications (2.7\%): two cases of acute post-ERCP pancreatitis (2\%) and one significant bleeding episode that was resolved by endoscopic sclerosis with adrenalin and ethoxysclerol, and which required red cell concentrate transfusion $(0.7 \%)$. All complications were controlled with medical and endoscopic treatment, except the perforation with stone impaction in the distal choledochus, which required emergency surgery. Globally, the number of complications was very low and similar in both groups, with no statistically significant differences between them (p 0.65) (Table 4). On grouping by type of complication, only acute pancreatitis reached a sufficient number to allow comparisons to be made. The Fisher exact test yielded $\mathrm{p}$ $=1$, i.e., in this case there likewise were no significant differences between the two patient groups (Table 5).

There were no deaths attributable to either technique 
Table 3. Inferential analysis. Comparison of success of stone removal in ES + SP and ES groups. There are no significant differences between the 2 groups $(\mathrm{p}=0.409)$.

\begin{tabular}{|c|c|c|c|c|}
\hline & & \multicolumn{2}{|c|}{ Satisfactory result } & \multirow{2}{*}{ Total } \\
\hline & & Yes & No & \\
\hline \multirow{4}{*}{ Case or control } & $\mathrm{N}$ & 49 & 3 & 52 \\
\hline & $\%$ & $94.2 \%$ & $5.8 \%$ & $100.0 \%$ \\
\hline & $\mathrm{N}$ & 98 & 3 & 101 \\
\hline & $\%$ & $97.0 \%$ & $3.0 \%$ & $100.0 \%$ \\
\hline \multirow{2}{*}{ Total } & $\mathrm{N}$ & 147 & 6 & 153 \\
\hline & $\%$ & $96.1 \%$ & $3.9 \%$ & $100.0 \%$ \\
\hline
\end{tabular}

Inferential analysis. Fisher test; $\mathrm{p}=0.409$; Nonsignificant $(\mathrm{p}>0.05)$.

Tabe 4. Inferential analysis. Comparison of global complications (pancreatitis, bleeding and perforation) in ES + SP and ES groups. There are no significant differences between the 2 groups $(\mathrm{p}=0.65)$.

\begin{tabular}{|c|c|c|c|c|}
\hline & & \multicolumn{2}{|c|}{ Global complications } & \multirow{2}{*}{ Total } \\
\hline & & Yes & No & \\
\hline \multirow{4}{*}{ Case or control } & $\mathrm{N}$ & 2 & 50 & 52 \\
\hline & $\%$ & $3.8 \%$ & $96.2 \%$ & $100.0 \%$ \\
\hline & $\mathrm{N}$ & 3 & 98 & 101 \\
\hline & $\%$ & $2.7 \%$ & $97.3 \%$ & $100.0 \%$ \\
\hline \multirow{2}{*}{ Total } & $\mathrm{N}$ & 5 & 148 & 153 \\
\hline & $\%$ & $3.28 \%$ & $96.72 \%$ & $100.0 \%$ \\
\hline
\end{tabular}

Table 5. Inferential analysis. Comparison of post-ERCP pancreatitis in ES + SP and ES groups. There are no significant differences between the 2 groups $(\mathrm{p}=1.000)$.

\begin{tabular}{|c|c|c|c|c|}
\hline & & \multicolumn{2}{|c|}{ Pancreatitis } & \multirow{2}{*}{ Total } \\
\hline & & Yes & No & \\
\hline \multirow{4}{*}{ Case or control } & $\mathrm{N}$ & 1 & 51 & 52 \\
\hline & $\%$ & $1.9 \%$ & $98.1 \%$ & $100.0 \%$ \\
\hline & $\mathrm{N}$ & 2 & 99 & 101 \\
\hline & $\%$ & $2.0 \%$ & $98.0 \%$ & $100.0 \%$ \\
\hline \multirow{2}{*}{ Total } & $\mathrm{N}$ & 3 & 150 & 153 \\
\hline & $\%$ & $2.0 \%$ & $98.0 \%$ & $100.0 \%$ \\
\hline
\end{tabular}

Inferential analysis. Fisher test; $\mathrm{p}=1.000$; Nonsignificant $(\mathrm{p}>0.05)$.

(ES with SP or ES alone).

Analysis of the possible influence of patient age or dilatation balloon diameter upon the appearance of complications showed no relationship between these variables and morbidity associated with the technique.

\section{DISCUSSION}

In most cases, biliary duct stones can be removed by ERCP with ES, using a Dormia basket of Fogarty balloons [9]. However, in the presence of large or numerous stones, or when the papilla does not allow extensive ES, the usual technique is proved to be unable to extract the stones in up to $15 \%$ of the cases $[3,4,10]$. Under these circumstances, we may use mechanical lithotripsy (involving shock waves, laser or electrohydraulics) or to opt for surgery [10]. Mechanical lithotripsy devices are not always easy to handle, and tend to significantly prolong the procedure. In addition, they are less effective in application to large or impacted stones, and have a relatively high complication rate $[5,9,10]$. More sophisti- 
cated intraductal lithotripsy techniques such as electrohydraulic or laser lithotripsy, etc., for the fragmentation of large stones, are expensive and available in very few centers [11-14]. The placement of plastic biliary stents may offer a temporary solution prior to surgical removal, or may represent a definitive treatment option in very elderly patients or individuals with a very high surgical risk $[14,15]$. When we use this technique and have already performed ES, we usually place more than one plastic stent in order to ensure improved drainage, and also to avoid displacements.

ES with SP is a very good alternative that minimizes the need for lithotripsy and surgery. The technique enlarges the diameter of the papilla and distal choledochus quickly and easily, and allows us to remove stones involving greater extraction difficulties. We maintain dilatation for approximately 30 seconds with each balloon caliber, and do not use more than three different diameters. With progressive balloons, the procedure can be carried out using a single balloon. The minimum diameter used in our experience was $10 \mathrm{~mm}$, with a maximum of $18 \mathrm{~mm}$. We found no relationship between the size of the balloon and the appearance of complications. There is no consensus in the literature on the extent of previous ES, dilatation time, or maximum balloon size. Shim et al. recommend a small ES in order to lessen the risk of perforation [14], while in contrast Attam and Mayedeo recommend that ES should be as extensive as possible $[11,16]$. We are more in agreement with this latter position, and do not modify the extent of ES according to whether dilatation will be carried out or not. Moreover, in many cases the decision to dilate is made after confirming that the stones cannot be removed with ES alone.

Martín-Arranz maintains insufflation during one minute [9], while other authors limit this time to between 15 - 30 seconds [13,17]. Most studies, including our own, use balloon diameters of between 10 and $20 \mathrm{~mm}[6,9,11$, $13,17-20]$, and in abidance with our own practice, it is generally advised not to exceed the diameter of the biliary tract [11]. We indistinctly use Fogarty balloons or Dormia baskets, and sometimes combine both devices. The risk of trapping within the papilla is greater with the basket, but traction force is greater. With the Fogarty balloon, trapping does not occur, but in some cases this device proves less effective.

In contrast to Itoi et al. [13], in our study the incidence of choledocholithiasis with extraction difficulties (multiple stones, large stones, peri-ampullar diverticulum or previous ES) was significantly greater in the ES with SP group than in the patients only subjected to ES. This is logical, considering that patient assignment to one group or others was not randomized. The addition of SP to ES was decided by the endoscopist, in those cases where removal of the stone or stones was expected to be difficult with the conventional methods, or when extraction with simple ES proved unsuccessful. This way of assigning patients to one group or others distinguishes our series from most published studies, in which distribution is made on a random basis. In this context, our study is more consistent with routine clinical practice, where treatment decisions are not taken randomly but depend on the circumstances of each individual case.

Although a priori removal of the stones proved more difficult in the ES with SP group, the success rate was similar in both cases (94\% in the ES with SP group versus $97 \%$ in the ES group) and consistent with the findings of other studies [13,14,16,17,19,21,22]. Likewise, no differences were observed between the two groups in terms of complications.

In the reviewed literature, the most common complication of ERCP with ES is acute pancreatitis (observed in $5 \%$ of all cases), followed by significant digestive bleeding (2\%), cholangitis (1\%) and perforation (0.3\%), the mean mortality rate being $0.4 \%[23,24]$. A larger number of complications would be expected on adding SP to ES, which has its own iatrogenic effects. This is all the more so when considering the greater complexity of those cases in which both procedures are combined. In our series, the global mortality rate was $3.28 \%$, and was only slightly higher in the ES with SP group (3.8\%) than in the ES group (2.7\%) - with no significant differences between them. Our complication rate was somewhat lower than in other studies $[13,14,16,17,22]$. The incidence of acute pancreatitis was practically identical in both groups (1.9\% in the ES with SP group versus $2 \%$ in the ES group), and in no case did we use pancreatic stents. This appears to confirm the findings in the literature to the effect that dilatation after ES results in far lower pancreatitis rates than when only performing SP $[13,17,25,26]$. Probably, the inflammatory reaction caused by SP, which can affect drainage of the pancreatic duct, is mitigated by the effect of previous ES, which separates the pancreatic and biliary orifices. Accordingly, to date SP without prior ES is reserved for situations in which ES is contraindicated, such as patients with coagulation disorders or receiving treatment with anticoagulants or antiplatelet drugs, etc. $[25,26]$. However, a recently published metaanalysis has concluded that SP involving longer dilatation (up to 5 minutes) and using larger diameter balloons is more effective, with effects similar to those of ES and involving similar pancreatitis and lower overall complication rates [27].

Our only case of perforation occurred in a patient with stenosis of the distal choledochus, and we considered that this complication was more a consequence of maneuvering during stone removal than a result of dilatation as such. Perforation was diagnosed from the identification of a retro-pneumoperitoneum on the abdominal X- 
rays. The isolated presence of a retro-pneumoperitoneum not associated with pneumoperitoneum usually implies a small perforation that normally can be managed with absolute diet, fluid therapy and antibiotics [28]. However, in our case the stone was impacted due to the lesser caliber of the distal choledochus and despite SP, as a result of which the decision was made to deal with both problems surgically.

In conclusion, endoscopic sphincterotomy with sphincteroplasty is a simple, safe and effective procedure for the management of choledocholithiasis with extraction difficulties, and reduces the need for other more aggressive, complex or expensive techniques.

\section{REFERENCES}

[1] Kawai, K., Akasaka, Y., Murakami, K., Tada, M. and Koli, Y. (1974) Endoscopic sphincterotomy of the ampula of water. Gastrointestinal Endoscopy, 20, 148-151. doi:10.1016/S0016-5107(74)73914-1

[2] Classen, M. and Demling, L. (1974) Endoscopic sphincterotomy of the papilla of water and extraction of stones from the choledochal duct. Dutch Med Wochenschr, 99, 496-497. doi:10.1055/s-0028-1107790

[3] Henry, L. and Lehman, G. (2006) Difficult bile duct stones. Current Treatment Options in Gastroenterology, 9, 123132. doi:10.1007/s11938-006-0031-6

[4] Hochberger, J., Tex, S., Maiss, J. and Hahn, E.G. (2003) Management of difficult common bile duct stones. Gastrointestinal Endoscopy Clinics of North America, 13, 623-634. doi:10.1016/S1052-5157(03)00102-8

[5] Stefanidis, G., Viazis, N., Pleskow, D., Manolakopoulos, S., Theokaris, L. and Cristodolou, C. (2011) Large baloon vs mechanical lithotripsy for the management of large bile duct stones: A prospective randomized study. American Journal of Gastroenterology, 106, 278-285. doi:10.1038/ajg.2010.421

[6] Ersoz, G., Tekesin, O., Osutemiz, A.O. and Gunsar, F. (2003) Biliary sphincterothomy plus dilation with large baloon for bile duct stones that are difficult to extract. Gastrointestinal Endoscopy, 57, 156-159. doi:10.1067/mge.2003.52

[7] Cotton, P.B. (1972) Cannulation of the papila of vater by endoscopic and retrograde cholangiopancreatography (ERCP). Gut, 13, 1014-1025. doi:10.1136/gut.13.12.1014

[8] Cotton, P.B. (1977) ERCP. Gut, 18, 316-341. doi:10.1136/gut.18.4.316

[9] Martín-Arranz, E., Rey-Sanz, R., Martín Arranz, M.D., Gea-Rodríguez, F., Mora-Sanz, P. and Segura-Cabral, J.M. (2012) Safety and efficacy of large baloon sphincteroplasty in a third care hospital. Revista Espanola de Enfermedades Digestivas, 102, 355-359. doi:10.4321/S1130-01082012000700004

[10] Wang, W.H., Chu, C.H., Wang, T.E., Chen, M.J. and Lin, C.C. (2005) Outcome of simple use of mchanical lithotripsy of difficult common bile duct stones. World Journal of Gastroenterology, 11, 593-596.
[11] Attam, R. and Freeman, M.L. (2009) Endoscopic papillary large baloon dilation for large common bile duct stones. Journal of Hepato-Biliary-Pancreatic Surgery, 16, 618-623. doi:10.1007/s00534-009-0134-2

[12] Itoi, T. and Wang, H.P. (2010) Endoscopic management of bile duct stones. Digestive Endoscopy, 22, 69-75. doi:10.1111/j.1443-1661.2010.00953.x

[13] Itoi, T., Itokawa, F., Sofuni, A., Kurihara, T., Tsuchiya, T., Ishii, K., et al. (2009) Endoscopic sphincterothomy combined with large baloon dilation can reduce the procedure time and fluoroscopy time for removal of large bile duct stones. American Journal of Gastroenterology, 104, 560-565. doi:10.1038/ajg.2008.67

[14] Shim, C.S. (2010) How should biliary Stone be managed? Gut and Liver, 4, 161-172. doi:10.5009/gnl.2010.4.2.161

[15] Nakayama, H.A., Kajiyama, M., Kato, N., Kamijima, T., Graham, D.Y. and Tanaka, N. (2010) Biliary stenting and the management of large or multiple common bile duct stones. Gastrointestinal Endoscopy, 71, 1200-1203. doi:10.1016/j.gie.2009.12.055

[16] Maydeo, A. and Bhandari, S. (2007) Baloon sphincterothomy for removing difficult bile duct sotones. Endoscopy, 11, 956-961.

[17] Kim, T.H., Oh, H.S., Lee, J.Y. and Sohn, Y. (2011) Can endoscopic sphincterothomy plus a large baloon dilation reduce the use of mechanical lithotripsy in patients winth large bile duct stones? Surgical Endoscopy, 25, 33303337. doi:10.1007/s00464-011-1720-3

[18] Espinel, J., Pinedo, E. and Olcoz, J.L. (2007) Large hydrostatic baloon for choledocholitiasis. Revista Espanola de Enfermedades Digestivas, 99, 33-38.

[19] Misra, S.L. and Dwivedi, M. (2008) Large-diameter baloon dilation after endoscopic sphincterotomy for removal of difficult bile duct stones. Endoscopy, 40, 209213. doi:10.1055/s-2007-967040

[20] Mirami, A., Shinji, H., Mamoto, T. and Hayakwa, S. (2007) Small sphincterotomy combined with paillary dilation with large baloon permits retrieval of large stones without mechanical lithotripsy. World Journal of Gastroenterology, 13, 2172-2182.

[21] Draganov, P.V., Evans, W., Fazel, A. and Forsmark, C.E. (2009) Large size baloon dilation of the ampula after biliary sphincteotomy can facilitate endoscopic extraction of difficult bile duct stones. Journal of Clinical Gastroenterology, 43, 782-786. doi:10.1097/MCG.0b013e31818f50a2

[22] Kurita, A., Maguchi, H., Takahashi, K., Katamura, A. and Osonai, M. (2010) Large baloon dilation for the treatment of recurrent bile duct stones in patients with previous sphincterotomy. Preliminary results. Scandinavian Journal of Gastroenterology, 45, 1242-1247. doi:10.3109/00365521.2010.495420

[23] Freeman, M.L., Di Sario, J.A., Nelson, D.B., et al. (2001) Risk factors for post ERCP pancreatitis: A prospective multicenter study. Gastrointestinal Endoscopy, 54, 425434. doi:10.1067/mge.2001.117550

[24] Freeman, M.L., Nelson, D.B., Sherman, S., et al. (1996) Complications of endoscopic biliary sphincterotomy. New England Journal of Medicine, 335, 909-918. 
doi:10.1056/NEJM199609263351301

[25] Fujita, N., Maguchi, H., Komatsu, Y., et al. (2003) Endoscopic sphincterotomy and endoscopic baloon dilation for bile duct stones: A prospective randomized controlled multicenter trial. Gastrointestinal Endoscopy, 57, 151155. doi:10.1067/mge.2003.56

[26] Di Sario, J.A., Freeman, M.L., Bjorkman, D.J., et al. (2004) Endoscopic baloon dilation compared winth sphincterotomy for extraction of bile duct stones. Gastroenterology, 127, 1291-1299. doi:10.1053/j.gastro.2004.07.017

[27] Chien, K.L., Tu, Y.K., Wu, M.S., Wang, H.P., Lin, J.T.,
Leung, J.W. and Chien, K.L. (2012) Baloon dilation with adequate duration is safer tan sphincterotomy for extracting bile duct stones: A sistematic review and meta-analyses. Clinical Gastroenterology and Hepatology, 10, 10091011.

[28] Machado, N.O. (2012) Management of duodenal perforation post-endoscopic retrograde cholangiopancreatography. When and whom to operate and what factors determine the outcome. A review article. Journal of the Pancreas, 13, 18-25.

\section{ABBREVIATIONS}

ERCP: endoscopic retrograde cholangiopancreatography ES: endoscopic sphincterotomy

SP: sphincteroplasty

$\mathrm{RR}$ : relative risk

$\mathrm{CI}$ : confidence interval

SD: standard deviation 